Journal of Pediatric Gastroenterology and Nutrition, Publish Ahead of Print DOI : 10.1097/MPG.0000000000002179

\title{
Sustainable positive response to sirolimus in juvenile polyposis of infancy
}

Muriel Quaranta ${ }^{1}$, Nolwenn Laborde ${ }^{1,2 *}$, Audrey Ferrand ${ }^{1}$, Marie Danjoux ${ }^{3}$,

Nathalie Vergnolle ${ }^{1}$, Frédérick Barreau ${ }^{1}$, Claire Racaud-Sultan ${ }^{1}$, Emmanuel Mas ${ }^{1,2}$

${ }^{1}$ IRSD, Université de Toulouse, INSERM, INRA, ENVT, UPS, Toulouse, France

${ }^{2}$ Unité de Gastroentérologie, Hépatologie, Nutrition, Diabétologie et Maladies Héréditaires du Métabolisme, Hôpital des Enfants, CHU de Toulouse, F-31300, France

${ }^{3}$ Département d'Anatomie et de Cytologie pathologiques, IUCT Oncopole, Toulouse, F-31300,

France

* Equal contributors

\section{Corresponding author:}

Emmanuel Mas, MD, PhD

Unité de Gastroentérologie, Hépatologie, Nutrition, Diabétologie et Maladies Héréditaires du

Métabolisme

330, avenue de Grande-Bretagne

TSA 70034

31059 Toulouse cedex 9

France

Tel. (33) 534558445

Fax. (33) 534558567 
E-mail: mas.e@chu-toulouse.fr

Financial support: Emmanuel Mas received a grant from the Rotary Club of Portet sur Garonne; INSERM

\section{Conflicts of interest and source of funding: none}

Keywords: juvenile polyposis syndrome; MTOR protein, human; PTEN protein, human; BMPR1A protein, human

Juvenile polyposis of infancy (OMIM 612242) 
Juvenile polyposis of infancy (OMIM 612242) is a hamartomatous polyposis including macrocephaly, facial dysmorphism, hypotonia, developmental delay; rectal bleeding occurred during the first 2 years of life $(1,2)$. It results from a microdeletion of chromosome 10q23 involving PTEN (phosphatase and tensing homolog) and BMPR1A (bone morphogenetic protein receptor type 1A) tumour-suppressor genes $(1,2)$. A protein-losing enteropathy can lead to colectomy in the first years of life $(1,2)$ and to an early death (2). Later on, there is an increased risk of colorectal cancer (2).

In mice, the conditional deletion of Bmprla induces the development of numerous polyps mimicking human juvenile polyposis (3). BMP signalling promotes cell differentiation and thus balances the intestinal stem cell renewal under the control of the Wnt/Apc/ -catenin pathway. In adenomatous polyposis coli $(A p c)$ deficient mice, the inducible loss of Pten leads to an increased tumourigenesis because of an increased activation of PI3K/Akt/mTOR (phosphatidyl-inositol 3 kinase/Protein kinase B/mammalian target of rapamycin) pathway (4).

mTOR is a downstream effector of the PI3K negatively regulated by PTEN. Its expression is increased in intestinal polyps of $A p c$ deficient mice; its inhibition reduced the number of polyps and their size (5), and also delayed the apparition of dysplasia (6). The wider use of the mTOR inhibitor sirolimus in clinical trials of cancer therapy and the severity of the phenotype of our young patient led us to consider this drug as a new therapeutic option.

An 11-year old girl was followed since birth for macrocephaly ( $+5 \mathrm{SD})$, mild hypotonia, motor and developmental delay. When she was 18 months old, she had rectal bleeding. Her colonoscopy revealed numerous juvenile polyps, up to $3 \mathrm{~cm}$ of diameter (Figure $1 \mathrm{~A}, \mathrm{~B}$ ). Genetic analysis found a de novo germline deletion encompassing PTEN and BMPR1A. Follow-up 
endoscopies confirmed a severe form of polyposis with 30 to 50 polyps, mainly located in the colon but also in the stomach and the small bowel. She had a failure to thrive, mild anaemia and protein-losing enteropathy. A colonic perforation occurred after resection of large polyps when she was 5 years old. In emergency, a transient ileostomy was performed.

Instead of performing a colectomy, which could exacerbate watery and stool losses and increase the risk of dehydration, we decided to start a treatment with sirolimus when she was 6-year-old, targeting a blood concentration of $5 \mathrm{ng} / \mathrm{mL}$. We found a decrease in number and size of polyps during yearly endoscopic follow-up (Figure $1 \mathrm{C}, \mathrm{D}$ ). After more than 4-year of follow-up, she showed a weight improvement and normal serum albumin level without recurrence of anaemia or adverse event. When she was 11-year-old no polyp was detected, even in the rectum where few small polyps were previously present.

Importantly, an immunostaining of phospho-S6 ribosomal protein, a target of mTOR, showed a marked decreased expression after sirolimus initiation (Figure 2).

In conclusion, this report suggests that sirolimus is effective in the treatment of severe juvenile polyposis of infancy and could be considered as a therapeutic option, instead of colectomy. 


\section{References}

1 Delnatte C, Sanlaville D, Mougenot JF, et al. Contiguous gene deletion within chromosome arm 10q is associated with juvenile polyposis of infancy, reflecting cooperation between the BMPR1A and PTEN tumor-suppressor genes. Am J Hum Genet 2006;78:1066-74.

2 Menko FH, Kneepkens CM, de Leeuw N, et al. Variable phenotypes associated with 10q23 microdeletions involving the PTEN and BMPR1A genes. Clin Genet 2008;74:14554.

3 He XC, Zhang J, Tong WG, et al. BMP signaling inhibits intestinal stem cell self-renewal through suppression of Wnt-beta-catenin signaling. Nat Genet 2004;36:1117-21.

4 Marsh V, Winton DJ, Williams GT, et al. Epithelial Pten is dispensable for intestinal homeostasis but suppresses adenoma development and progression after Apc mutation. Nat Genet 2008;40:1436-44.

5 Fujishita T, Aoki K, Lane HA, et al. Inhibition of the mTORC1 pathway suppresses intestinal polyp formation and reduces mortality in ApcDelta716 mice. Proc Natl Acad Sci U S A 2008;105:13544-9.

6 Hardiman KM, Liu J, Feng Y, et al. Rapamycin inhibition of polyposis and progression to dysplasia in a mouse model. PLoS One 2014;9:e96023. 
Figure 1: Juvenile polyps under sirolimus treatment. Prior to treatment, there were large pediculated or sessile polyps with exudative loss on their heads (A, B). The number and size of polyps decreased after 1 and 3 years of treatment (C and D respectively).
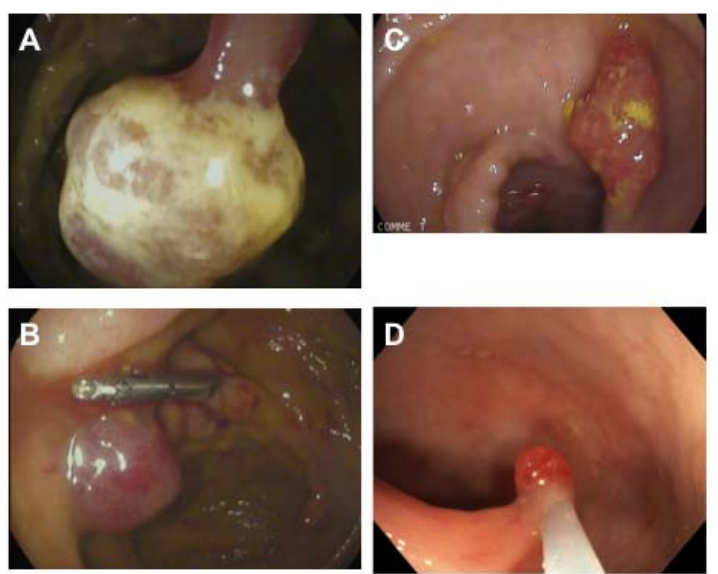
Figure 2: mTOR activity in sirolimus-treated polyps.

Immunostaining of phospho-S6 ribosomal protein (left panels) was performed to measure mTOR activity under sirolimus treatment. Nuclei staining (DAPI, right panels) and the whole sections (boxes) of the polyps are shown. Despite a strong and large decrease in phospho-S6 ribosomal protein labelling, demonstrating mTOR inhibition, some polyps areas remained positive (see box). 